\title{
Advances in the 3-D Forward-Backward Time-Stepping (FBTS) Inverse Scattering Technique for Breast Cancer Detection
}

\author{
Jessi E. Johnson*, Member, IEEE, Takashi Takenaka, Member, IEEE, Kismet Anak Hong Ping, \\ Shunsuke Honda, and Toshiyuki Tanaka
}

\begin{abstract}
This paper presents recent advances in a 3-D inverse scattering technique, called forward-backward time-stepping (FBTS), applied to the reconstruction of the microwave properties of the breast. The FBTS algorithm is utilized for a numerical-based study of a 3-D breast model based on an MRI. Several illumination schemes, based on different microwave transmitter/receiver configurations, are compared based on the quality of the reconstructed images of the breast model. A combination of cylindrical and planar arrays is shown to provide accurate estimates of the model electrical parameters that delineate the various regions of the breast. Although further analysis with this combination array demonstrates that tumors of reduced size and reduced contrast with the surrounding fibroglandular region are much more difficult (and in some cases not possible) to reconstruct, the study presents some promising initial results of a reconstruction technique for breast imaging and cancer detection.
\end{abstract}

Index Terms-Cancer, electromagnetic scattering inverse problems, medical diagnosis, microwave imaging.

\section{INTRODUCTION}

A CTIVE microwave breast imaging, in which microwave signals are transmitted into the breast with subsequent scattered energy utilized to analyze the breast's composition and detect the presence or absence of malignancies, has been developing rapidly over the past several years. The motivation behind the development of this technology stems from the fact that the current standard for breast cancer detection, $\mathrm{X}$-ray mammography, is limited in terms of both sensitivity (correctly detecting breast cancer) and specificity (correctly screening out patients without malignancies) [1].

The potential for microwave imaging for practical clinical use is significant due to a wide variety of possible advantages such as low-cost system implementation, the use of low-power, nonionizing radiation [2], [3], patient comfort [4], and promising initial clinical investigations [4], [5]. Another advantage frequently cited in the literature, a large contrast in the electrical

Manuscript received May 5, 2008; revised October 22, 2008. First published May 19, 2009; current version published August 14, 2009. This work was supported in part by a Japan Society for the Promotion of Science (JSPS) Grant-inAid for Scientific Research(B) (20360187) and a Grant-in-Aid for JSPS Fellows (17-05901). Asterisk indicates corresponding author.

* J. E. Johnson was with the Department of Electrical and Electronic Engineering, Nagasaki University, Nagasaki 852-8521, Japan. He is now with Miramar Labs, Inc., Sunnyvale, CA 94085 USA (e-mail: johnsonj@ieee.org).

T. Takenaka, K. A. H. Ping, S. Honda, and T. Tanaka are with the Department of Electrical and Electronic Engineering, Nagasaki University, Nagasaki 852-8521, Japan (e-mail: takenaka@nagasaki-u.ac.jp).

Digital Object Identifier 10.1109/TBME.2009.2022635 properties of tumors and the surrounding breast tissue in the microwave frequency range, has recently been shown to be less drastic than previously imagined [6]. This new understanding of breast tissue composition poses a new and difficult challenge for the microwave-based approach to imaging.

Recent advances in active imaging include syntheticaperture-radar-(SAR) based techniques such as tissue sensing adaptive radar (TSAR) [7]-[9] and microwave imaging via space-time beamforming (MIST) [10]-[12], both of which have demonstrated the ability to detect tumors both in numerical studies and realistic phantom experiments. Another approach is the time-reversal technique [13] in which signals acquired from scattering measurements of the breast are back propagated to achieve a focusing effect on a scatterer (i.e., tumor) in the breast, potentially leading to detection and localization. Additionally, frequency-domain inverse scattering approaches such as those of Meaney and coworkers have also demonstrated successful numerical and phantom results [14], [15], in addition to very promising preliminary clinical studies [5], [16].

The forward-backward time-stepping (FBTS) technique is an approach to microwave imaging in which a nonlinear inverse scattering computation is formulated in the time domain utilizing finite-difference time-domain (FDTD) simulation to reconstruct the dielectric properties of an object. This approach was reported by Takenaka et al. [17] for a simple 1-D case, and has been extended to reconstruction of simple objects in 2-D [18], [19] and 3-D for both numerical- [20] and measurementbased studies [21], and more recently, to a heterogeneous breast model in 2-D [22], [23]. Similar time-domain approaches utilizing an FDTD-based approach have also been presented in the literature [24]-[27], including a recent 2-D investigation of a heterogeneous breast model that included dispersive effects [26] and a measurement-based 2-D study of simple dielectric objects [27]. The FBTS technique allows a broad spectrum of frequencies to be utilized in a single optimization, giving the potential for a large amount of information about the internal composition of the breast to be obtained. Additionally, the use of a nonlinear inverse scattering approach, although computationally intensive, allows quantitative reconstruction of the electrical properties of the breast to be obtained, rather than the detection of scatterers as is the case for radar-based approaches. This has the potential advantage of allowing discrimination between malignancies and other relatively high-water-content tissues such as fibroglandular tissue. In this study, an extension of the FBTS technique to a 3-D numerical study of a heterogeneous 


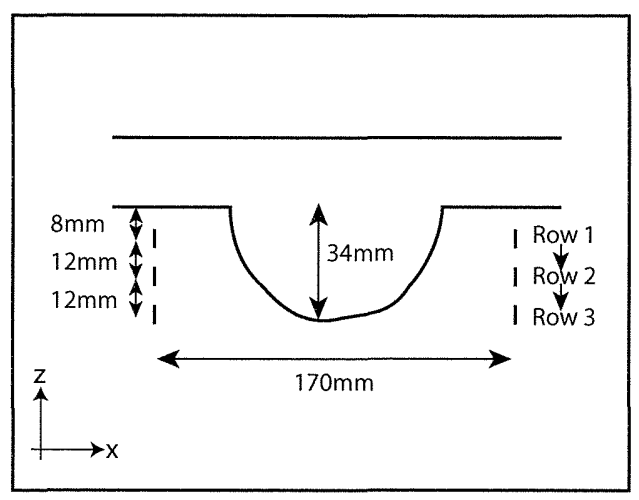

(a)

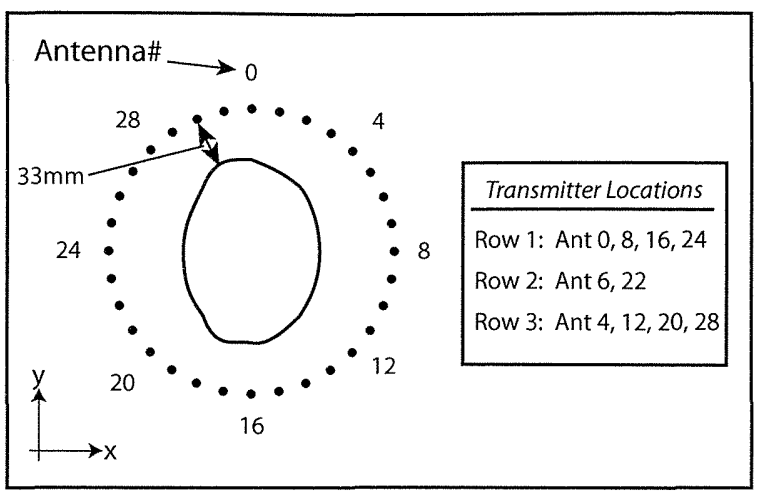

(b)

Fig. 1. Block diagram showing the vertically scanned circular scattering characterization scheme. (a) $x z$ plane, showing the three vertical measurement positions to which the circular array is scanned. (b) $x y$ plane, showing the 32-element circular array and a tabulation of which antennas are utilized as transmitters at each vertical position (row).

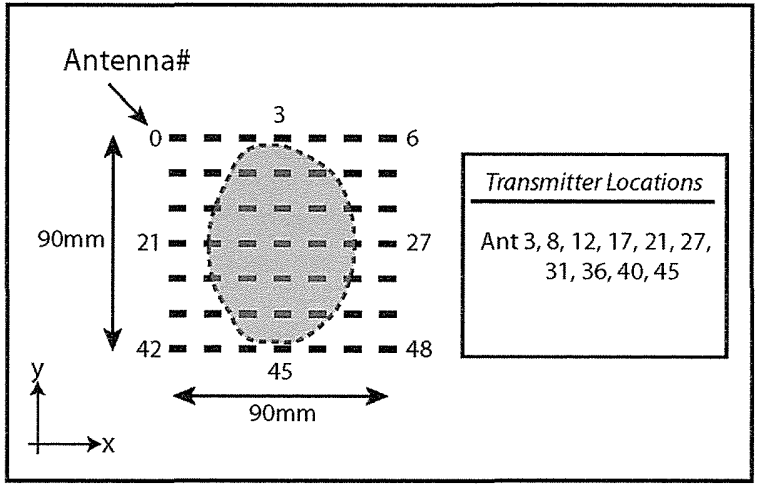

Fig. 2. Block diagram showing the planar scattering characterization scheme in the $x y$ plane. The 49 elements in the array are shown along with a tabulation of which antennas are utilized as transmitters.

MRI-derived breast model is presented, providing further preliminary evidence of the potential of the FBTS approach for breast cancer detection. First, in Section II, an overview of the FBTS imaging technique is provided. This is followed by details regarding the development of the breast model (see Section IIIA), the implementation of the algorithm (see Section III-B), and the scattering characterization schemes utilized (see Section IIIC). Results of the study are shown in Section IV, and followed by Section V, which provides some discussion and analysis of the results, shortcomings of the approach when applied to more difficult imaging problems, and areas of future development. Finally, Section VI gives a brief conclusion.

\section{FBTS IMAGING}

The foundation of the FBTS technique lays in the formulation of an error functional that compares measured and corresponding computed scattering data in the time domain. A multistatic scattering measurement system is utilized to characterize the breast, as demonstrated in the basic diagrams shown in Figs. 1-3 (described in further detail shortly). The breast is surrounded by an array of antennas and a set of measured scattering data is acquired by utilizing one antenna as a transmitter and receiving the subsequent scattered signal with the surrounding antennas. A different transmitting antenna is then selected and the process is repeated until a desired set of transmit/receive data for multiple antenna combinations is characterized. This measured dataset is compared to an equivalent simulation in which the same set of scattering data are computed for an assumed set of dielectric parameters $p$ representing the electrical property profile of the breast. The set of parameters $p$ can consist simply of the permittivity $\epsilon_{r}$ and conductivity $\sigma$, or can be a more complex representation such as a set of first-order Debye parameters [26]. In the FBTS approach, the computed scattered dataset is conveniently calculated in the time-domain utilizing the FDTD technique.

The error functional $Q(p)$ for the assumed set of dielectric parameters $p$ is expressed as

$$
Q(p)=\int_{0}^{T} \sum_{m=1}^{M} \sum_{n=1}^{N}\left|v_{m}\left(p ; r_{n}, t\right)-\tilde{v}_{m}\left(r_{n}, t\right)\right|^{2} d t
$$

where $\tilde{v}_{m}\left(r_{n}, t\right)$ represents the measured received scattered E-field data at a receiving position $n$ due to a transmitter $m$, and $v_{m}\left(p ; r_{n}, t\right)$ represents the corresponding computed scattered E-field data for the assumed set of parameters $p$. The errors between the measured and computed scattered fields are summed for each transmitter/receiver combination and integrated over the entire duration of the signal $(T)$.

By taking the Fréchet derivative [28] of (1) and introducing a set of electromagnetic fields $w_{m i}(p ; r, t)$ that represent the solution to the adjoint form of Maxwell's equations [17]-[20], the gradients of the error functional $Q(p)$ with respect to $\epsilon_{r}$ and $\sigma$ can be expressed by

$$
\begin{aligned}
& g_{\epsilon_{r}(r)}=\int_{0}^{T} \sum_{m=1}^{M} \sum_{i=1}^{3}\left[w_{m i}(p ; r, t) \frac{d}{d t} v_{m i}(p ; r, t)\right] d t \\
& g_{\sigma}(r)=\int_{0}^{T} \sum_{m=1}^{M} \sum_{i=1}^{3}\left[w_{m i}(p ; r, t) v_{m i}(p ; r, t)\right] d t
\end{aligned}
$$




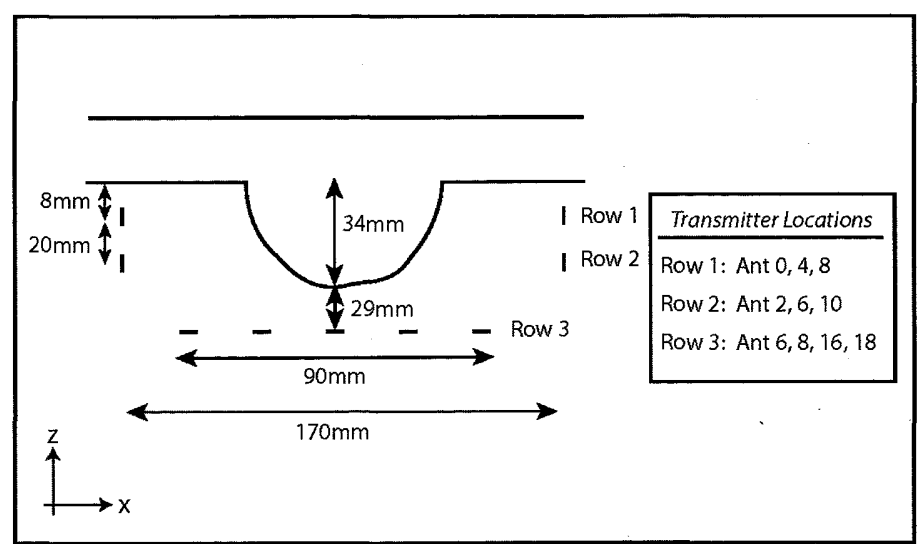

(a)

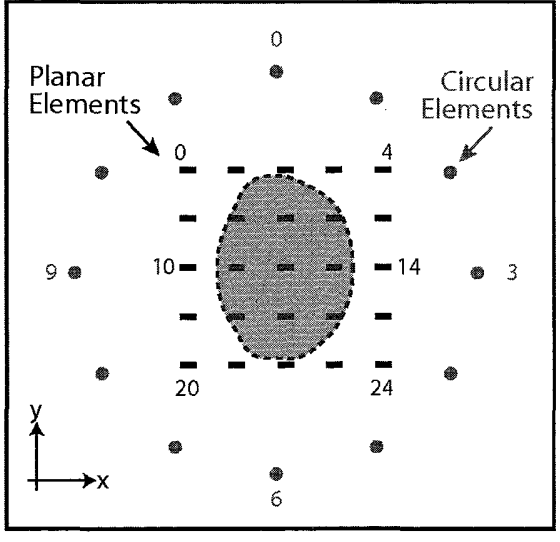

(b)

Fig. 3. Block diagram showing the combination scattering characterization scheme. (a) $x z$ plane, showing the vertical positions of the two circular arrays and the planar array, and a tabulation of which antennas are utilized as transmitters at each vertical position (row). (b) $x y$ plane, showing the 12 elements in the circular arrays, as well as the 25 elements in the planar array.

where the field values $v_{m i}(p ; r, t)$ and $w_{m i}(p ; r, t)$ are those calculated in the reconstruction region $r$. As described in [17] and [20], the adjoint field terms can also be computed utilizing the FDTD method, with the received residual scattered signals $\left[v_{m}\left(p ; r_{n}, t\right)-\tilde{v}_{m}\left(r_{n}, t\right)\right]$ being reversed in time and utilized as sources. The summation term from $i=1$ to $i=3$ represents the fact that in 3-D the gradients are calculated using the $x$-, $y$-, and $z$-components of $v_{m i}$ and $w_{m i}$ and then added together. The gradient terms (2) and (3) allow the conjugate gradient technique [29] to be utilized to minimize the error functional (1), resulting in an estimation of the electrical parameters $p$.

Note that in this study it is assumed that the media is dispersionless, with constant permittivity and conductivity over changes in frequency. This assumption allows for a less complex reconstruction algorithm where two $\left(\epsilon_{r}\right.$ and $\left.\sigma\right)$ rather than three (e.g., $\epsilon_{s}, \epsilon_{\infty}$, and $\sigma_{s}$ ) or more parameters need to be reconstructed, and is utilized for this early stage investigation.

\section{METHODS}

\section{A. MRI-Based Breast Modeling}

Building upon previous 2-D MRI-based breast modeling work described in [22], an anatomically realistic 3-D breast model has been developed. The modeling approach, which is very similar to the piecewise linear approach described in [30], consists of mapping the pixel intensities of an MR image of the breast to an equivalent set of Debye parameters. This is done by first plotting the histogram of the pixel intensities of the MR image and segmenting the image into three distinct regions including a fat region, fibroglandular region, and fat to fibroglandular transition region. Pixel intensity levels that delineate these regions are chosen by visual inspection of the MR image.

After performing the segmentation of the MR image, a piecewise linear mapping scheme is utilized to assign a set of Debye model parameters to each pixel intensity. Nominal Debye parameters from the literature [30] for fat and fibroglandular tissue are assigned to the pixel intensities that define their nominal intensity on the histogram. The nominal Debye values $\left(\epsilon_{s}, \epsilon_{\infty}\right.$, and $\sigma_{s}$ ) are then varied linearly by $\pm 10 \%$ within each tissue
TABLE I

ELECTRICAL PROPERTY PARAMETERS UtILIZED FOR BREAST MODEL

\begin{tabular}{lllllll}
\hline \hline Tissue & $\epsilon_{s}$ & $\epsilon_{\infty}$ & $\sigma_{s}$ & $\tau$ & \multicolumn{2}{l}{$\epsilon_{r 2 G H z} \sigma_{2 G H z}$} \\
\hline fibroglandular & 21.57 & 6.14 & 0.31 & $7.0 \mathrm{e}-12$ & 21.45 & 0.46 \\
fat & 10.00 & 7.00 & 0.15 & $7.0 \mathrm{e}-12$ & 9.98 & 0.18 \\
skin & 37.00 & 4.00 & 1.10 & $7.2 \mathrm{e}-12$ & 36.73 & 1.43 \\
tumor/chest wall & 54.00 & 4.00 & 0.70 & $7.0 \mathrm{e}-12$ & 53.62 & 1.19 \\
coupling liquid & - & - & - & - & 9.98 & 0.0 \\
\hline
\end{tabular}

region (with $\tau$ left constant). The values assumed for the various tissue types are summarized in Table I. It should be noted that the nominal values assumed for the fibroglandular tissue were significantly lower than indicated in the recent study by Lazebnik et al. [6]. As such, the contrast between the tumor and surrounding fibroglandular tissue is significantly higher than might typically be expected in practice. However, in this early stage investigation, this less challenging case was considered sufficient to quantify the efficacy of the 3-D FBTS technique in a significantly more complex heterogeneous situation than previously investigated. An initial analysis of a reduced contrast model was also conducted, as described in Section III-D shortly.

A 2-mm-thick skin layer and a 10-mm-diameter spherical tumor are added to the model and these pixels are assigned the nominal Debye parameters for their respective tissue types. Finally, since a nondispersive case was assumed in this study, the Debye parameters for the entire model are evaluated at the center frequency of the input signal used in subsequent FDTD simulations ( $2 \mathrm{GHz}$ in this case), resulting in a final, anatomically accurate breast permittivity and conductivity model. Since this study utilizes a nondispersive assumption, a simpler modeling approach in which constant permittivity and conductivity values are mapped directly is also possible. However, taking the approach of utilizing a Debye-based model allows for future expansion to a dispersive case.

\section{B. Algorithm Setup}

As described earlier, the FBTS algorithm utilizes FDTD simulation for solving both the forward scattering problem as well 
as the adjoint field computation. A custom FDTD subroutine was implemented in Fortran, with the convolutional perfectly matched layer (CPML) formulation [31] used to terminate the simulation boundaries. The FBTS algorithm was implemented in the Python programming language, which allowed high-level scripting commands to be utilized to define the scattering characterization scheme and drive the FDTD module. One of the biggest challenges in moving from a 2-D to 3-D implementation of the FBTS algorithm is the increased computational demand. Being an iterative, FDTD-based technique, reconstruction times can quickly become prohibitively large when moving to a 3-D case. Thus, the FBTS algorithm was implemented with a parallel computing structure and utilized message passing interface (MPI) commands in Python to distribute the calculations to multiple processors.

Numerical scattered signal datasets were formed using FDTD simulations of the MRI-based breast model on a $1 \mathrm{~mm} \times 1 \mathrm{~mm} \times$ $1 \mathrm{~mm}$ grid with a $2 \mathrm{GHz}$ sinusoidally modulated Gaussian pulse with a $1.3 \mathrm{GHz}$ bandwidth. This frequency range was chosen to give an optimal tradeoff between the resolution of the image and the convergence of the imaging algorithm [27]. Antennas were modeled as discrete $50 \mathrm{ohm}$ line sources extending across a single gridline in the FDTD space. This simplified antenna model allowed the desired pulse to be utilized without distortion due to the antenna response (e.g., that of a narrowband dipole antenna). Eventual integration of the FBTS technique with real measured data will require precise modeling of the antenna and will be subject to the limitations in the antenna performance, and thus will be a focus of future development. Alignment of the antennas relative to the breast is detailed in Section III-C shortly.

The FBTS computations were conducted assuming that the breast was measured in a lossless coupling liquid with relative permittivity similar to the nominal value assumed for fat tissue $\epsilon_{r}=9.98$. The choice of an immersion liquid roughly matching breast fat was demonstrated to be efficient for imaging in previous studies [22], [32], although it is also a variable for further optimization. For the reconstructions, it was also assumed that the position of the breast and thickness of the skin could be detected. This is a fairly large assumption made for this early stage investigation, although microwave-based approaches are under development both for breast position [33], [34] and skin thickness estimation [35].

An initial permittivity and conductivity value of $+10 \%$ of the nominal value was assumed for the skin, and the remaining "normal" breast tissue region was assigned dielectric values equal to the coupling liquid $\left(\epsilon_{r}=9.98, \sigma=0.0\right)$. Pixels in the entire breast region, including the skin region, were allowed to vary during the reconstruction with pixels outside the breast region fixed as equal to the coupling liquid. The reconstructions were carried out for 100 iterations for all cases, which although not explicitly optimized, ensured that only minimal decreases in the error functional were observed for subsequent iterations ( $2 \%$ or smaller, with exception of the planar case described shortly). Calculation time was $38 \mathrm{~min}$ per iteration utilizing a parallel computing cluster of ten PCs with AMD Athlon $644000+$ processors and 4 GB of RAM.

\section{Scattering Characterization Schemes}

In order to assess the efficacy of the 3-D FBTS approach, the MRI-derived breast model was analyzed utilizing four illumination schemes. These consisted of a vertically scanned circular configuration, a cylindrical configuration, a planar configuration, and a combination cylindrical/planar configuration. Each of the four configurations utilized either circular arrays of antennas, a planar array of antennas, or a combination of both. Circular array elements were aligned to radiate a $z$-polarized E-field, while planar elements were aligned to radiate an $x$-polarized field. A minimum antenna to breast distance of between 29 and $33 \mathrm{~mm}$ was utilized, which approached the closest allowable distance before the antennas exhibited reactive coupling that would cause inaccuracies in the reconstruction. For each of the configurations, ten total transmitting locations were utilized and all available receivers were utilized for each transmitter. The total number of antennas for each configuration was chosen such that a similar number of $\mathrm{Tx} / \mathrm{Rx}$ combinations could be utilized, with the exception of the vertically scanned case. Details of each of the configurations are provided below.

1) Vertically Scanned Circular: The first configuration consists of a $170 \mathrm{~mm}$ diameter circular array of 32 antennas aligned in a single plane, as shown in Fig. 1. In this configuration, a set of scattering measurements is taken with the array aligned in a vertical position $8 \mathrm{~mm}$ from the chest wall (row 1). The array is then moved down $12 \mathrm{~mm}$ in the negative $z$-direction to row 2 and another set of measurements is made, and the process is repeated once again at row 3. Fig. 1 shows which antenna elements were utilized as transmitters for each row, these locations were selected such that the breast was illuminated from a variety of angles in the $x y$-plane encircling the breast in a symmetric pattern. In this configuration, a total of $310 \mathrm{Tx} / \mathrm{Rx}$ combinations are used. This configuration contains significantly fewer scattering data (fewer $\mathrm{Tx} / \mathrm{Rx}$ combinations) than the other cases; however, it is a practical case (see for example [4]) that warranted analysis and comparison to the cases with more $\mathrm{Tx} / \mathrm{Rx}$ combinations.

2) Cylindrical: The cylindrical configuration consists of three rows of 16-element circular arrays (170 $\mathrm{mm}$ in diameter). Rows are spaced $12 \mathrm{~mm}$ from each other, and the row closest to the chest wall is $8 \mathrm{~mm}$ away. The configuration is equivalent to the configuration shown in Fig. 1, except there are three rows of antennas present (rather than a single scanned row) and there are only half the number of antennas in each row. The transmitter locations in each row are the same as for the vertically scanned case. For each transmitter there are 47 receivers, thus giving a total of $470 \mathrm{Tx} / \mathrm{Rx}$ combinations.

3) Planar: The planar configuration consists of a 49element array (on a $7 \times 7$ grid) that spans $90 \mathrm{~mm}$ in either direction, as shown in Fig. 2. Elements are spaced $15 \mathrm{~mm}$ from each other in the $x$ - and $y$-directions, and are aligned to radiate an $x$-polarized E-field. The array was placed at a vertical distance of $63 \mathrm{~mm}$ from the chest wall and $29 \mathrm{~mm}$ from the closest point on the breast. Transmitting positions are detailed in Fig. 2 and were chosen to radiate signals into the breast from a variety of angles in a symmetric pattern in the $x y$ plane. For each 


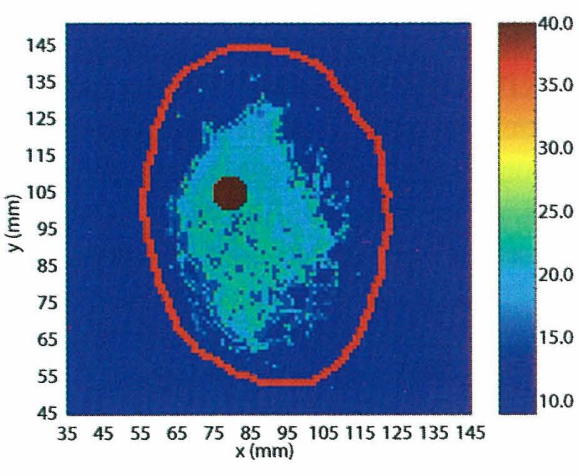

(a)

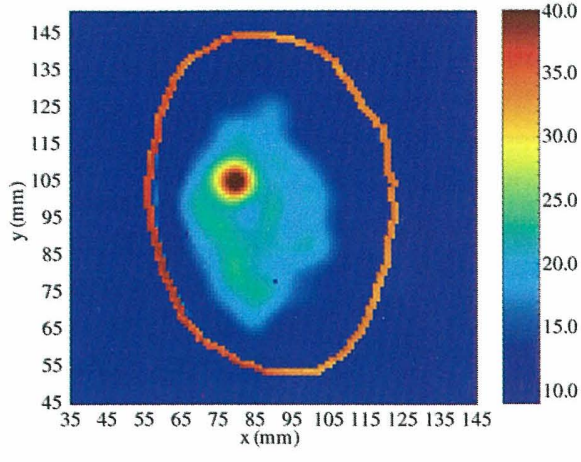

(b)

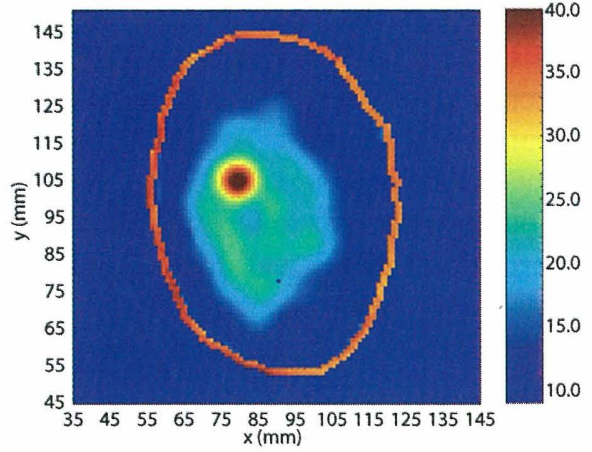

(c)

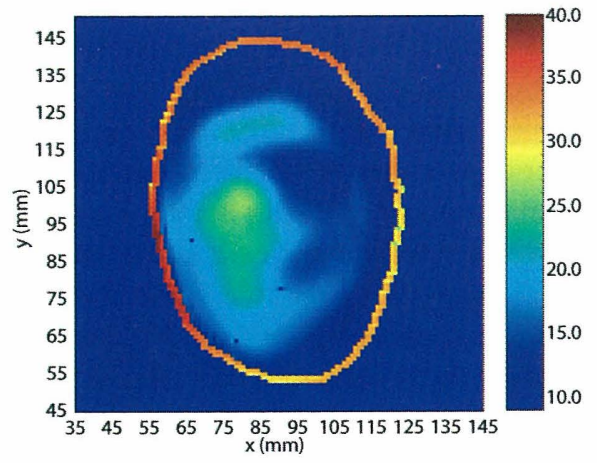

(d)

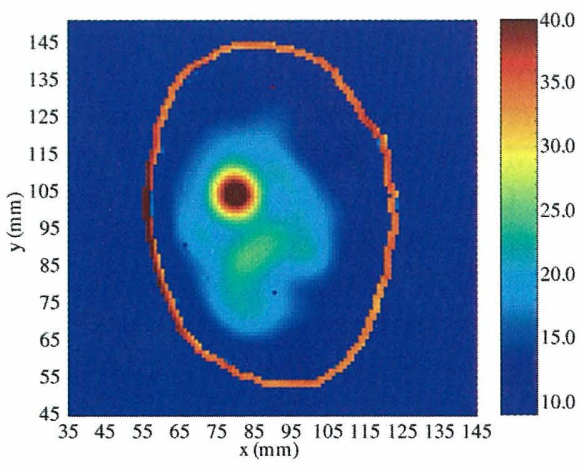

(e)

Fig. 4. Permittivity images in $x y$ plane $(z=60 \mathrm{~mm}$ ) of breast model with $10 \mathrm{~mm}$ tumor in the fibroglandular region: (a) model, (b) vertically scanned configuration, (c) cylindrical configuration, (d) planar configuration, and (e) combination configuration.

transmitter there are 48 receivers, thus giving a total of 480 $\mathrm{Tx} / \mathrm{Rx}$ combinations.

4) Combination: The combination configuration consists of two rows of 12-element circular arrays (170 $\mathrm{mm}$ in diameter), as shown in Fig. 3. The rows are spaced $20 \mathrm{~mm}$ from each other, and the row closest to the chest wall is $8 \mathrm{~mm}$ away. This configuration also includes a 25 -element planar array (on a $5 \times 5$ grid) that spans $90 \mathrm{~mm}$ in either direction. The transmitter locations were selected using the same methodology as for the previous cases. For each transmitter there are 48 receivers, giving a total of $480 \mathrm{Tx} / \mathrm{Rx}$ combinations. This configuration which combines cylindrical and planar arrays is similar to the scheme presented in [34].

\section{Reduced Contrast and Tumor Size}

In addition to the analysis described earlier, effects of reduced contrast and tumor size on the efficacy of the FBTS technique were analyzed. The effects of reduced tumor volume were demonstrated by reconstructing models with an $8 \mathrm{~mm}$ tumor and a $5 \mathrm{~mm}$ tumor using the same breast model properties presented in Section III-A. Effects of reduced contrast were also studied using a breast model with nominal fibroglandular tissue properties derived from recent studies from Lazebnik et al. [6], [36] demonstrating higher permittivity and conductivity values than previously shown. Specifically, a reduced contrast model was created by utilizing the "group 2" breast tissue, single-pole Debye model from [36] for the fibroglandular region (with $\epsilon_{s}=$ $37.65, \epsilon_{\infty}=5.57, \sigma_{s}=0.52, \tau=8.68 \mathrm{e}-12, \epsilon_{r 2 \mathrm{GHz}}=37.27$, $\left.\sigma_{2 \mathrm{GHz}}=0.91\right)$. The values for the other regions were identical to the model of Table I and a $10 \mathrm{~mm}$ diameter tumor was utilized. For both the reduced size tumor models and the reduced contrast model, reconstructions were conducted using the combination configuration and compared to the reconstruction of the original model with the $10 \mathrm{~mm}$ tumor.

\section{RESULTS}

Fig. 4. shows a 2-D horizontal cross section (in the $x y$ plane) through the 3-D permittivity reconstructions for each of the illumination schemes described earlier, with the model included for comparison. Reconstruction of the tumor, as well as the surrounding fibroglandular region is clearly demonstrated for the vertically scanned, cylindrical, and combination cylindricalplanar array illumination schemes. The peak permittivity values in the tumor region are $\epsilon_{r}=45.6$ for the vertically scanned configuration, $\epsilon_{r}=49.54$ for the cylindrical configuration, and $\epsilon_{r}=45.9$ for the combination configuration compared to the actual value of $\epsilon_{r}=53.62$. The planar configuration reconstruction [see Fig. 4(d)] is of significantly reduced quality compared with the other schemes, with the tumor indistinguishable from the surrounding tissue. Corresponding vertical 2-D cross sections (in the $x z$ plane) for the permittivity reconstructions are shown in Fig. 5. While the vertically scanned, cylindrical, and combination configuration images are relatively similar in the horizontal cross section, some distinct differences are exhibited in the vertical cross section. The shape of the reconstructed tumor for the vertically scanned based image [see Fig. 5(b)] is 


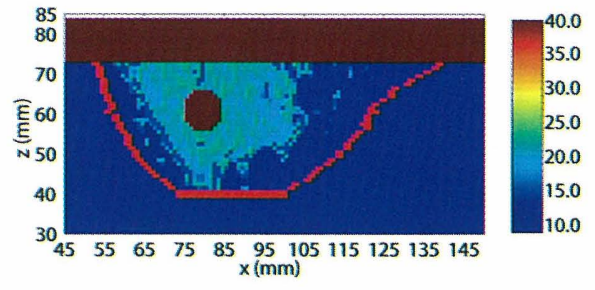

(a)

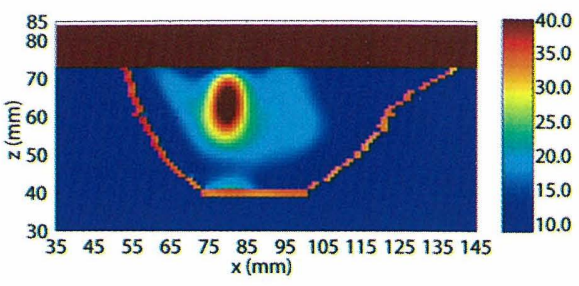

(b)

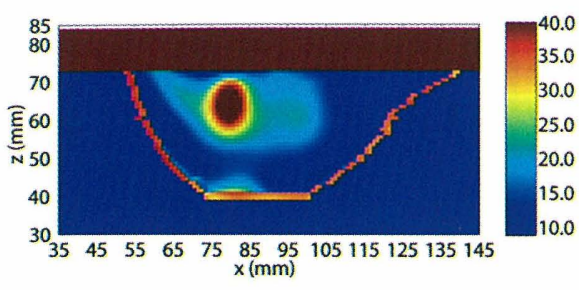

(c)

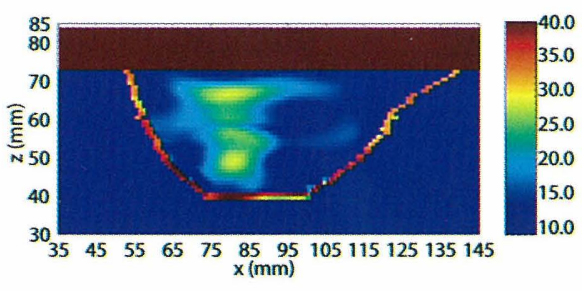

(d)

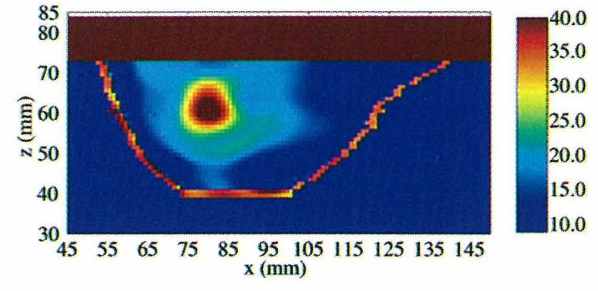

(e)

Fig. 5. Permittivity images in $x y$ plane $(y=104 \mathrm{~mm}$ ) of breast model with $10 \mathrm{~mm}$ tumor in the fibroglandular region: (a) model, (b) vertically scanned configuration, (c) cylindrical configuration, (d) planar configuration, and (e) combination configuration.

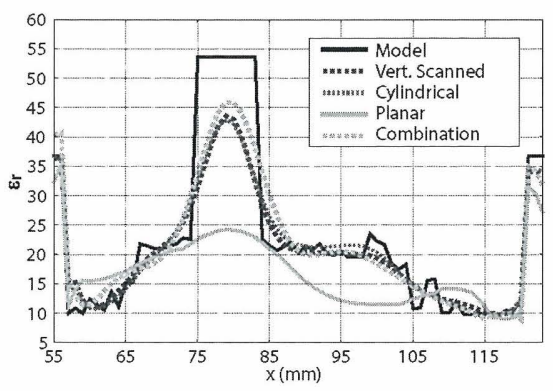

(a)

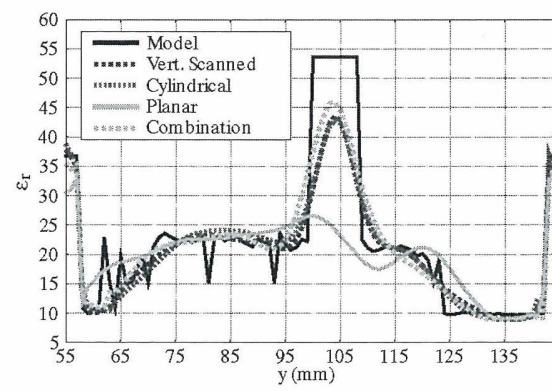

(b)

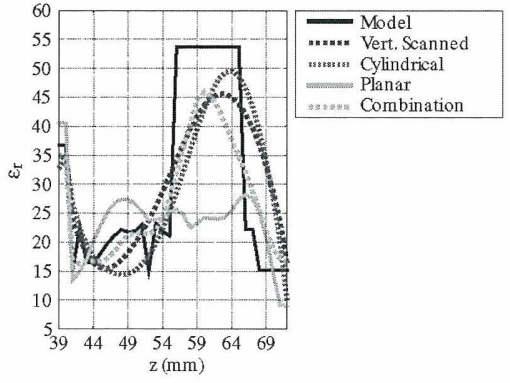

(c)

Fig. 6. 1-D cuts through 3-D permittivity reconstructions: (a) $x$-axis $(y=104 \mathrm{~mm}, z=60 \mathrm{~mm})$, (b) $y$-axis $(x=79 \mathrm{~mm}, z=60 \mathrm{~mm})$, (c) $z$-axis $(x=79 \mathrm{~mm}$, $y=104 \mathrm{~mm})$.

stretched vertically and appears somewhat cylindrical. In contrast, the cylindrical and combination configurations reconstruct a tumor with a more circular cross section that better approximates the modeled lesion. Again, for all three cases (vertically scanned, cylindrical, and combination), the fibroglandular region is reconstructed distinctly from the fat region. Fig. 5 also demonstrates the reduced image quality when the planar configuration is utilized.

Fig. 6. shows 1-D cuts through the 3-D permittivity images along the $x$-, $y$-, and $z$-axis on a line cutting through the center of the tumor (which is positioned at $x=79 \mathrm{~mm}, y=104 \mathrm{~mm}$, and $z=60 \mathrm{~mm}$ ) and provides a quantitative comparison of the four illumination schemes. Accurate tumor detection (for all cases except the planar configuration) is demonstrated by both the large predicted permittivity value in the tumor region, as well as the accurate prediction of the tumor position-particularly for the combination configuration. The accuracy of the permittivity reconstructions is further quantified in Table II. The table shows the averaged permittivity and conductivity values for the fibroglandular, fat, and skin regions of the initial model and reconstructed images (determined utilizing pixels at sets of $x$, $y, z$-coordinates for the various tissue regions defined in the model). Observing first the fibroglandular region, it is seen that
TABLE II

QUANTITATIVE COMPARISON OF PERMITTIVITY AND CONDUCTIVITY RECONSTRUCTIONS

\begin{tabular}{|c|c|c|c|c|c|c|}
\hline . & & Model & $\begin{array}{l}\text { Vert- } \\
\text { Scan }\end{array}$ & $\begin{array}{l}\text { Cylin- } \\
\text { drical }\end{array}$ & Planar & $\begin{array}{l}\text { Combi- } \\
\text { nation }\end{array}$ \\
\hline \multirow{4}{*}{ Fibro } & $\epsilon_{a v g}$ & 21.5 & 18.9 & 19.0 & 17.2 & 19.1 \\
\hline & $\sigma_{a v g}(\mathrm{~S} / \mathrm{m})$ & 0.46 & 0.44 & 0.38 & 0.47 & 0.43 \\
\hline & $\epsilon_{a v g}(\%$ err $)$ & & -12.1 & -11.8 & -20.2 & -11.3 \\
\hline & $\sigma_{a v g}(\%$ err $)$ & & -4.4 & -17.7 & 2.9 & -6.3 \\
\hline \multirow{4}{*}{ Fat } & $\epsilon_{\text {avg }}$ & 10.1 & 11.2 & 11.2 & 11.7 & 11.1 \\
\hline & $\sigma_{a v g}(\mathrm{~S} / \mathrm{m})$ & 0.18 & 0.21 & 0.22 & 0.24 & 0.20 \\
\hline & $\epsilon_{\text {avg }}(\%$ err $)$ & & 11.0 & 10.9 & 15.8 & 10.1 \\
\hline & $\sigma_{\text {avg }}(\%$ err $)$ & & 15.5 & 21.5 & 32.0 & 13.2 \\
\hline \multirow{4}{*}{ Skin } & $\epsilon_{a v g}$ & 36.7 & 34.3 & 34.3 & 34.1 & 34.9 \\
\hline & $\sigma_{a v g}(\mathrm{~S} / \mathrm{m})$ & 1.43 & 1.32 & 1.35 & 1.25 & 1.33 \\
\hline & $\epsilon_{\text {avg }}(\%$ err $)$ & & -6.5 & -6.6 & -7.2 & -4.9 \\
\hline & $\sigma_{a v g}(\%$ err $)$ & & -7.4 & -5.9 & -12.3 & -6.7 \\
\hline \multirow{6}{*}{ Tumor } & $\epsilon_{\text {avg }}$ & 53.6 & 35.6 & 36.2 & 23.4 & 36.5 \\
\hline & $\sigma_{a v g}(\mathrm{~S} / \mathrm{m})$ & 1.19 & 0.68 & 0.60 & 1.17 & 0.94 \\
\hline & $\epsilon_{\text {avg }}(\%$ err $)$ & & -33.7 & -32.5 & -56.4 & -31.9 \\
\hline & $\sigma_{a v g}(\%$ err $)$ & & -42.2 & -49.2 & -1.2 & -21.1 \\
\hline & $\epsilon_{\Delta}$ cntr $(\mathrm{mm})$ & & 3.0 & 4.0 & 6.4 & 0.0 \\
\hline & $\sigma_{\Delta} \operatorname{cntr}(\mathrm{mm})$ & & 4.7 & 2.4 & 5.1 & 2.2 \\
\hline
\end{tabular}

the averaged permittivity values are most accurate for the combination configuration, having an $-11.3 \%$ error when compared with the model. The vertically scanned and cylindrical cases 


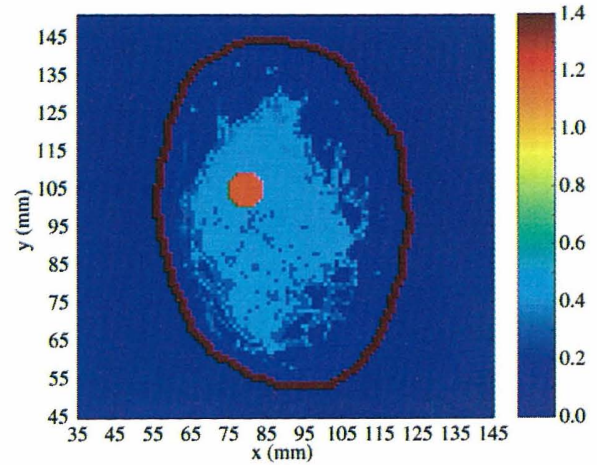

(a)

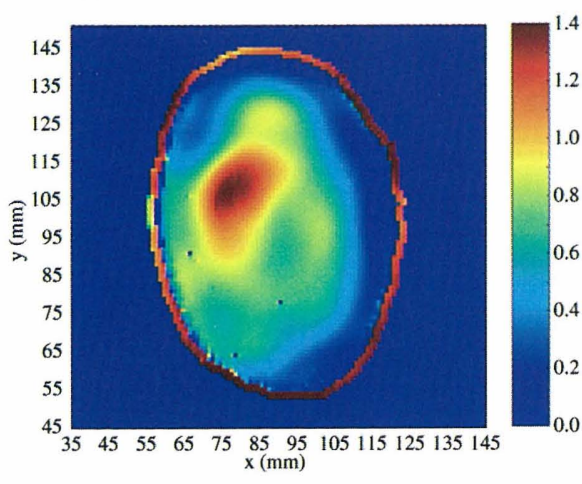

(d)

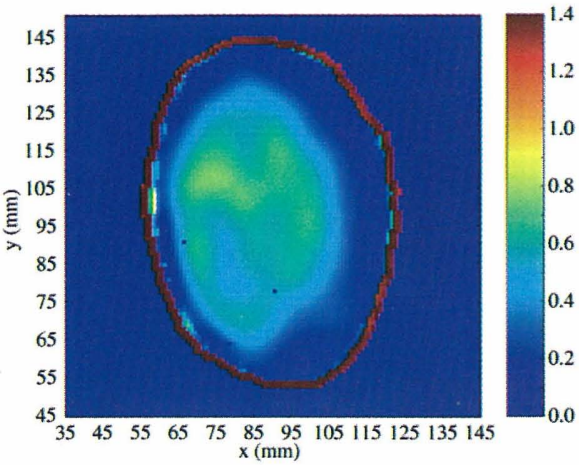

(b)

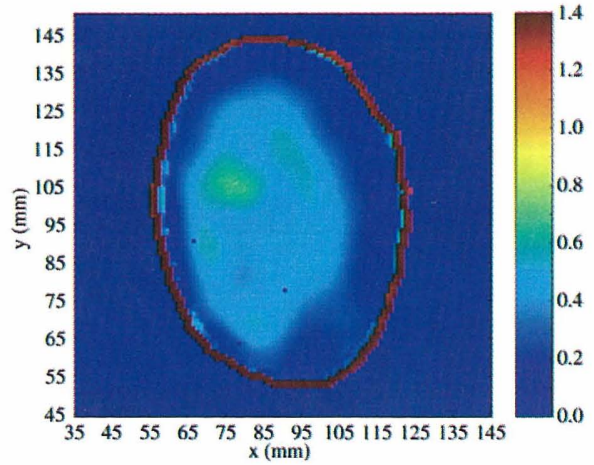

(c)

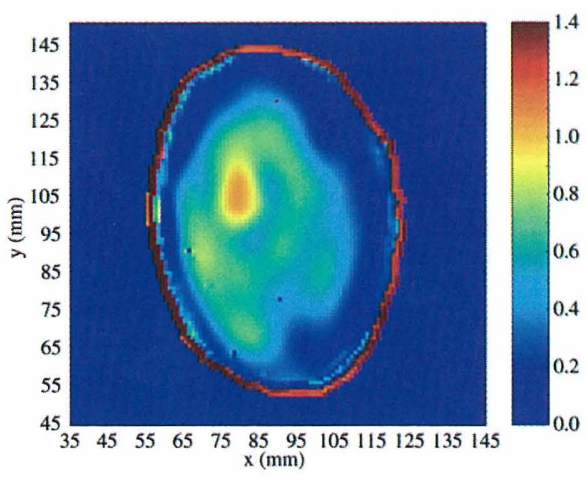

(e)

Fig. 7. Conductivity images in $x y$-plane $(z=60 \mathrm{~mm}$ ) of breast model with $10 \mathrm{~mm}$ tumor in the fibroglandular region: (a) model, (b) vertically scanned configuration, (c) cylindrical configuration, (d) planar configuration, and (e) combination configuration.

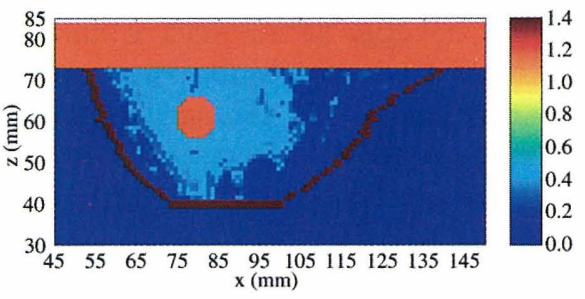

(a)

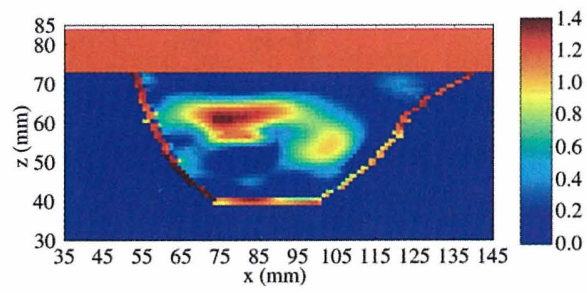

(d)

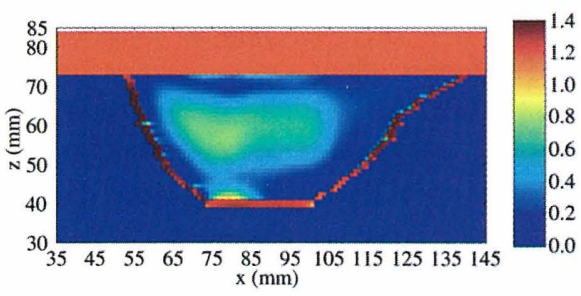

(b)

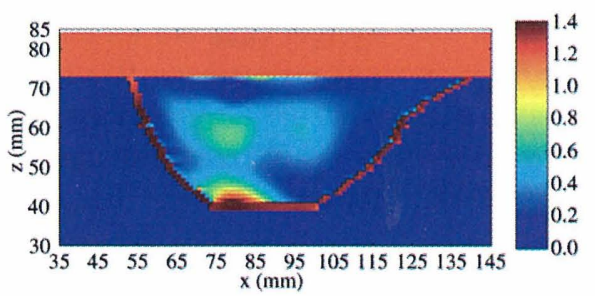

(c)

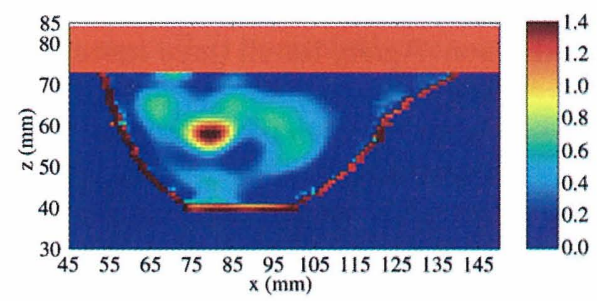

(e)

Fig. 8. Conductivity images in $x z$-plane $(y=104 \mathrm{~mm}$ ) of breast model with $10 \mathrm{~mm}$ tumor in the fibroglandular region: (a) model, (b) vertically scanned configuration, (c) cylindrical configuration, (d) planar configuration, and (e) combination configuration.

exhibit similar accuracy as the combination configuration while the planar configuration is significantly less accurate $(-20.2 \%$ error compared with the model). In the fat and skin regions, average permittivity values are similar for all four reconstructions. For the tumor region, as in the other regions, the average permittivity value is most accurate for the combination configuration. Table II also demonstrates the localization of the tumor, with the value $\epsilon_{\Delta}$ center showing the difference between the actual and predicted center point of the tumor (calculated for the recon- structions utilizing the $x, y$, and $z$-coordinate of the maximum permittivity value in the tumor region). The localization for the combination configuration matches the model exactly and the vertically scanned and cylindrical cases also predict the tumor position to within $4.0 \mathrm{~mm}$.

Figs. 7 and 8 . show the conductivity reconstructions corresponding to the permittivity reconstructions of Figs. 4 and 5 (with scaling renormalized to a scale from $\sigma=0$ to 1.4, rather than the permittivity scaling from $\epsilon_{r}=9$ to 40 ). Observing 


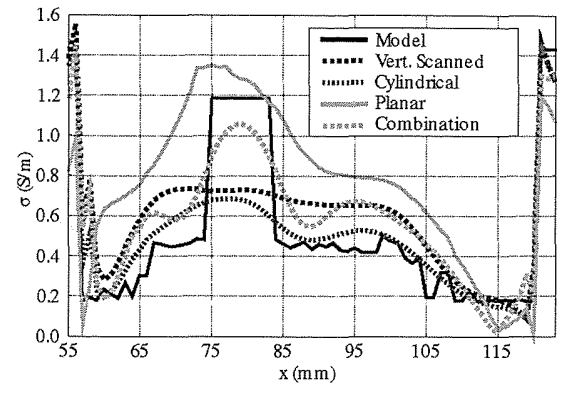

(a)

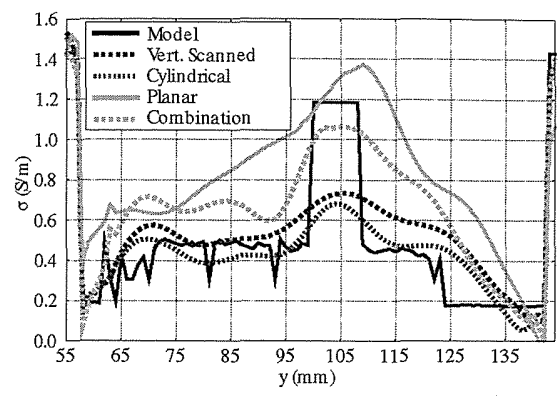

(b)

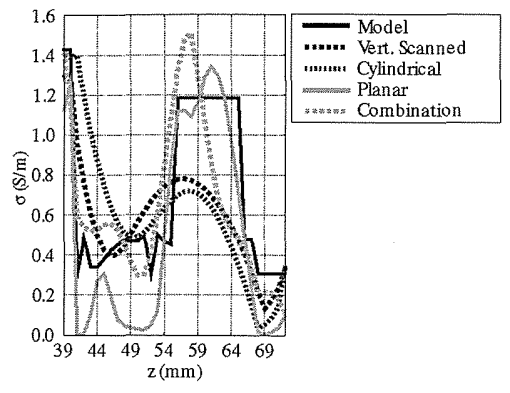

(c)

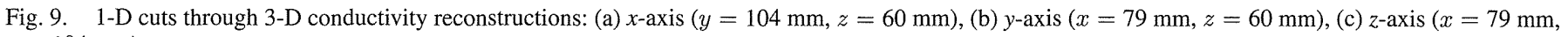
$y=104 \mathrm{~mm})$.

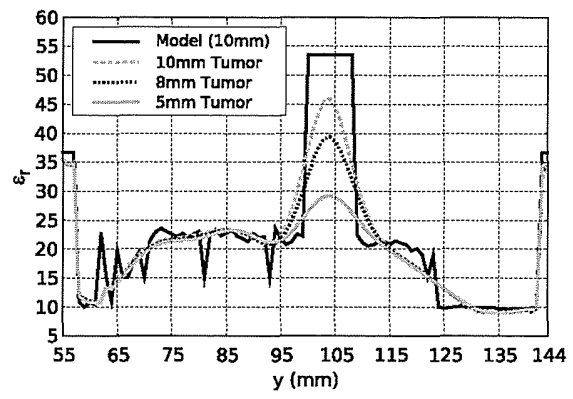

(a)

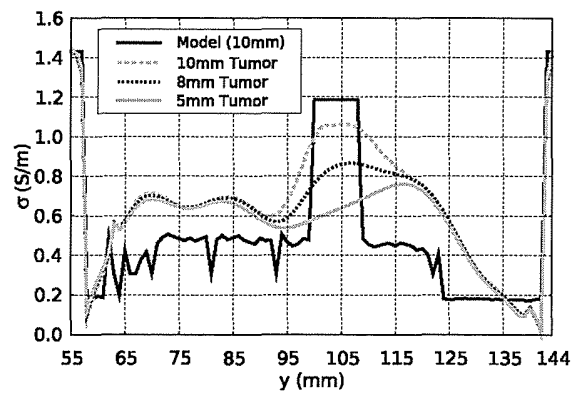

(b)

Fig. 10. 1-D cuts along the $y$-axis $(x=79 \mathrm{~mm}, z=60 \mathrm{~mm}$ ) through 3-D reconstructions with varying diameter tumors. (a) Permittivity image. (b) Conductivity image.

Fig. 7, it is clear that the conductivity images are of lower general quality than the corresponding permittivity images. The tumor does not appear distinctly in the conductivity image for the vertically scanned, cylindrical, or planar configurations. For the combination configuration [see Fig. 7(e)] the tumor is distinguishable from the surrounding fibroglandular tissue, although the contrast between the tumor and surrounding tissue is greatly reduced compared to the permittivity image. The shape of the reconstructed tumor for the combination configuration is also distorted, being stretched along the $y$-axis. The vertical cross sectional reconstructions of Fig. 8 demonstrate similar results as the horizontal cross sections. The combination configuration provides a reconstruction of the tumor, while the other cases fail to do so. However, the vertically scanned and cylindrical cases give a reasonably accurate reconstruction of the fibroglandular region distinct from the surrounding fat tissues. This is observed in both the horizontal and vertical planes.

Fig. 9. shows 1-D cuts through the conductivity images (as the same manner shown in Fig. 6 for the permittivity images). In contrast to the permittivity images, the reconstructions of the model are fairly poor for all of the illumination schemes except the combination configuration. The vertically scanned and cylindrical configurations result in reconstructions that generally track through the fat and fibroglandular regions fairly well particularly along the $x$ - and $y$-axis, as shown in Fig. 9(a) and (b); however, there are no significant increases in the tumor region indicating tumor detection. The combination configuration tracks relatively accurately through the fat and fibroglandular regions and rises to a local maximum that delineates the presence
TABLE III

COMPARISON OF RECONSTRUCTIONS OF VARYING DIAMETER TUMORS UTILIZING COMBINATION CONFIGURATION

\begin{tabular}{llllll}
\hline \hline & & Model & $10 \mathrm{~mm}$ & $8 \mathrm{~mm}$ & $5 \mathrm{~mm}$ \\
\hline \multirow{4}{*}{ Fibro } & $\epsilon_{\text {avg }}$ & 21.5 & 19.1 & 19.1 & 19.0 \\
& $\sigma_{\text {avg }}(\mathrm{S} / \mathrm{m})$ & 0.46 & 0.43 & 0.43 & 0.42 \\
\cline { 2 - 6 } & $\epsilon_{\text {avg }}(\%$ diff) & & -11.3 & -11.4 & -11.6 \\
& $\sigma_{\text {avg }}(\%$ diff) & & -6.3 & -7.0 & -8.9 \\
\hline \multirow{4}{*}{ Tumor } & $\epsilon_{\text {avg }}$ & 53.6 & 36.5 & 34.1 & 27.8 \\
& $\sigma_{\text {avg }}(\mathrm{S} / \mathrm{m})$ & 1.19 & 0.94 & 0.79 & 0.63 \\
\cline { 2 - 6 } & $\epsilon_{\text {avg }}(\%$ diff) & & -31.9 & -36.4 & -48.1 \\
& $\sigma_{\text {avg }}(\%$ diff) & & -21.1 & -33.5 & -46.8 \\
\cline { 2 - 6 } & $\epsilon_{\Delta \text { cntr }(\mathrm{mm})}$ & 0.0 & 0.0 & 1.0 \\
& $\sigma_{\Delta}$ cntr $(\mathrm{mm})$ & 2.2 & 2.2 & $*$ \\
\hline \multirow{2}{*}{ *Tumor not detected for this case. } & & &
\end{tabular}

of a tumor and approximates the tumor from the model. Table II shows further quantitative data for the conductivity reconstructions. From the table it is shown that the average reconstructed conductivity values are fairly similar for all four reconstructions for the fibroglandular, fat and skin regions. The distinct difference in the average conductivity values is shown in the average tumor values, which are superior for both the combination and planar cases (although, as demonstrated in Figs. 7 and 8, the general quality of the planar configuration image is poor).

Results for reconstructions of reduced size tumors with the combination configuration are shown in Fig. 10, which shows a 1-D plot comparing the model to the reconstructed results for the original $10-\mathrm{mm}$ diameter tumor, as well as the smaller diameter tumors. As might be expected, the image quality begins to deteriorate as the tumor diameter is decreased, with the 5-mm diameter tumor barely registering a permittivity level that is 


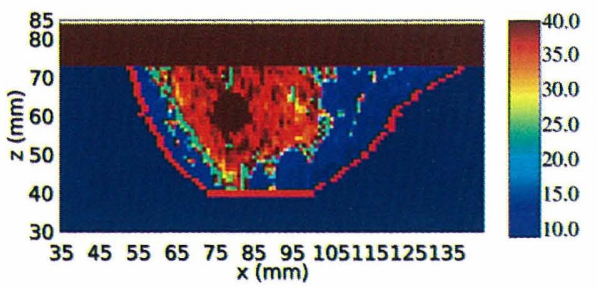

(a)

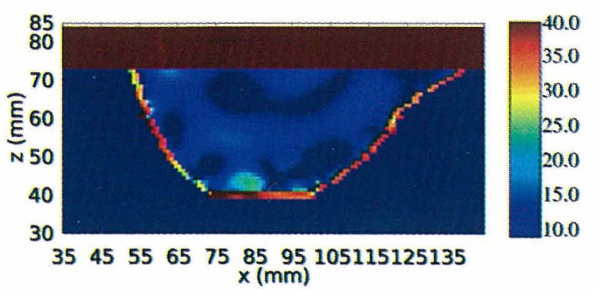

(b)

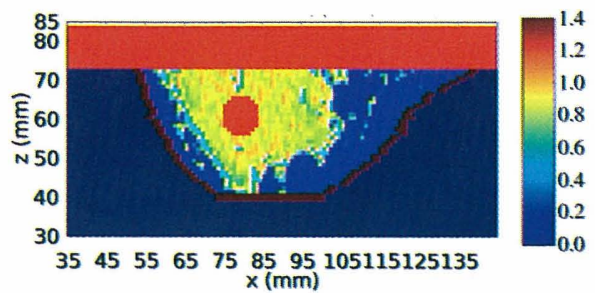

(c)

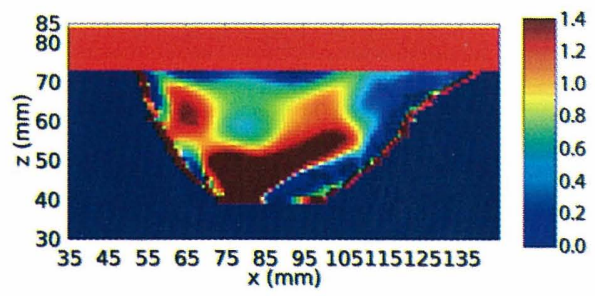

(d)

Fig. 11. Images in $x z$-plane $(y=104 \mathrm{~mm}$ ) of reduced contrast model with $10 \mathrm{~mm}$ tumor in the fibroglandular region: (a) permittivity model, (b) permittivity reconstruction, (c) conductivity model, and (d) conductivity reconstruction.

larger than the fibroglandular tissue in the area of the tumor [see Fig. 10(a)]. Additionally, the conductivity reconstruction for the 5-mm diameter tumor [see Fig. 10(b)] shows no distinguishable peaks in the region of the tumor. Table III provides a quantitative comparison of the reconstructed images and demonstrates that while the accuracy of the reconstruction of the fibroglandular region of the breast was virtually identical for all three cases (the skin and fat regions were also similar for all three cases but are not shown), the average permittivity and conductivity values in the tumor region begin to deviate from the modeled value as the tumor diameter decreases. Localization of the tumor is similar for all three tumors, with the exception of the conductivity reconstruction of the 5-mm diameter tumor, which was indistinguishable from the fibroglandular region.

Reconstructions of the reduced contrast model are demonstrated in Fig. 11, which shows the model and corresponding reconstructed $\epsilon_{r}$ and $\sigma_{s}$ images in the $x z$ plane. As the figure shows, the reduced contrast between the tumor and fibroglandular region had the effect of masking the tumor. Additionally, the algorithm had difficulty converging on an accurate solution to the fibroglandular region in general. This is clearly evident in Fig. 11(b), which shows that the reconstructed permittivity values in the fibroglandular region were much too low. For the reconstructed permittivity image the $\epsilon_{\mathrm{avg}}=11.9$ in the fibroglandular region compared to an $\epsilon_{\mathrm{avg}}=37.4$ for the model. In the fat region the permittivity reconstruction resulted in an $\epsilon_{\mathrm{avg}}=$ 11.2 compared to an $\epsilon_{\mathrm{avg}}=10.1$ for the model. Fig. 11(d) demonstrates that the conductivity image generally has larger conductivity values in the fibroglandular region than in the surrounding fat region, however the actual conductivity value is over-predicted, particularly in the lower region of the breast (between $z=40$ and $z=50$ ). For the reconstructed conductivity image the $\sigma_{\mathrm{avg}}=1.01$ in the fibroglandular region compared to a $\sigma_{\text {avg }}=0.91$ for the model. In the fat region the average conductivity was over-predicted significantly, with the $\sigma_{\text {avg }}=0.46$ for the image compared to a $\sigma_{\mathrm{avg}}=0.18$ for the model.

\section{Discussion}

The differences in image quality among the four illumination schemes examined in the numerical study provide some significant insight into the choice of measurement points for scattering characterization of the breast. The data clearly suggest, as might be expected, that illumination of the breast with a larger variety of vertical ( $z$-direction) and horizontal ( $x$ - and $y$-direction) angles gives better image quality for an equivalent number of transmitter/receiver combinations. The planar configuration, in which the antennas are aligned in a single plane ( $x y$-plane), results in very poor reconstruction quality compared to the other schemes. The reduced quality in this case is likely compounded by chest wall effects, with the tumor response being largely masked by large reflections from the chest wall, an effect described in [37].

The vertically scanned configuration utilizes three measurement planes and, as is particularly evident in Fig. 6, has greatly improved tumor detection compared to the planar reconstruction. This configuration is advantageous for practical implementation as a vertically scanned circular antenna array, as has been presented in the literature [5], [14]. As discussed in [38], however, a potential shortcoming of this approach is the fact that no "cross-plane" measurements are possible, with the consequence of less precise vertical resolution of a tumor. The reconstructions presented in this work reinforce this concept, with a slightly "stretched" tumor shape along the $z$-axis observed for the vertically scanned case, as shown in Figs. 5(b) and 6(c).

The combination configuration, like the cylindrical configuration, utilizes three measurement planes. However, it is clear in this case that combining cylindrical and planar elements has maximized the amount of information contained in the scattering data, resulting in a less ill-posed [39] inverse- scattering problem and subsequently an improved image quality. This is most evident by the fact that the combination configuration results in tumor detection for both the permittivity and conductivity images. Additionally, the combination configuration 
demonstrates improved accuracy in the permittivity reconstruction of the fibroglandular region near the chest wall. This is observed in comparison of the images of Fig. 5(b), (c), and (e) in the region between the tumor and chest wall. Finally, the results summarized in Table II further highlight the improvements in the permittivity and conductivity reconstructions provided by the combination configuration. Although for the most part the averaged $\epsilon_{r}$ and $\sigma$ values are similar in the various tissue regions, the reconstructed tumor conductivity is significantly more accurate for the combination configuration. The combination case also provides superior localization of the tumor for both the permittivity and conductivity reconstructions:

The numerical study also demonstrates that the reconstructed image quality is significantly greater for the permittivity than the conductivity. This is a known phenomenon that can be mainly attributed to the fact that the error functional (1) tends to be more sensitive to deviations in the permittivity than the conductivity. Small phase shifts in the time domain due to permittivity changes generally cause great differences between the measured and computed the scattered waveforms. Changes in the amplitude due to conductivity differences are less significant and thus become less heavily weighted in (1).

One assumption utilized in this study that warrants discussion was that the position of the breast could be detected and the skin thickness estimated. Although this assumes a fairly large amount of a priori information about the breast, microwave technology is under development to estimate the breast location [33], [34], the thickness of the skin [35], and the average breast tissue properties [26]. Although not analyzed in this work, it is also possible that less accurate estimates for the breast shape and position will also provide adequate image quality, as demonstrated in the 2-D study presented in [22]. Nonmicrowave approaches for conducting these measurements, for example optical positioning techniques [40] or skin thickness measurements via ultrasound [41], could certainly also be envisioned for future integration with the FBTS technique.

Finally, some discussion regarding future challenges for the FBTS approach is provided. Tumors of reduced size were clearly more difficult to detect, although some success was demonstrated even with a 5-mm diameter tumor. Reduced contrast models had deleterious effects on the FBTS algorithm, causing it to converge on an inaccurate solution with poor reconstruction quality. Although clearly there are limitations in the present implementation of the FBTS technique, future enhancements to the approach should be possible. As discussed in [27], in the iterative time-domain inverse scattering process there is a strong relationship between the center frequency of the pulse being utilized, resolvable object size, and stability of algorithm convergence. Lower frequency pulses lend themselves to resolving larger objects and tend to be more stable, while higher frequency pulses can improve resolution but may not converge on a valid solution. In [42] it was demonstrated that utilizing transmitted pulses of incrementally increasing frequency during iterations of the FBTS technique could overcome the effects of noisy data. A similar approach may allow low resolution images that delineate, for example, the fibroglandular region from areas of skin and adipose-dominated tissue to be created at lower-frequencies and utilized as initial guesses to stabilize subsequent higher frequency reconstructions for resolving smaller, lower contrast tumors. Other techniques, such as edge-preserving regularization [19], [43] or other regularization schemes may also possibly be incorporated to improve the FBTS algorithm accuracy and stability. Exploring such techniques is a critical area of further investigation and advancement of the FBTS approach. For future integration with measured scattering data, it is also possible that dispersive effects will need to be included as part of the FBTS algorithm. This is possible by utilizing, for example a first-order Debye model for the material properties and differentiating the error functional (1) with respect to the Debye parameters $\left(\epsilon_{s}, \epsilon_{\infty}\right.$, and $\left.\sigma_{s}\right)$. Preliminary success in conducting 2-D time-domain inverse scattering using a Debye model has been demonstrated in an approach similar to the FBTS technique by Winters et al. [26]. Development of scattering measurement systems and integration of measurement data with the FBTS technique is another future challenge for this approach.

\section{CONCLUSION}

In this study, recent advances in the 3-D FBTS inverse scattering technique for breast cancer detection have been presented. A numerical study based on scattering simulations of an MRIderived breast model provided preliminary evidence of the efficacy of the FBTS method for tumor detection and discrimination of the various internal breast tissue types in three dimensions. Although the technique has demonstrated promising results, it also had limited success resolving tumors of reduced size and contrast. Future development of this technique to address these issues, as well as integrate dispersive properties and practical measurement data should further increase the potential of FBTS for breast cancer detection.

\section{REFERENCES}

[1] Committee on Technologies for the Early Detection of Breast Cancer, Mammography and Beyond: Developing Technologies for the Early Detection of Breast Cancer, S. Nass, I. Henderson, and J. Lashof, Eds. Washington, DC: Nat. Cancer Policy Board, Inst. Med., Commission Life Stud, Nat. Res. Council, 2001.

[2] J. Johnson and T. Takenaka, "Preliminary assessment of electromagnetic absorption in the breast for cylindrical microwave breast cancer detection systems," in Proc. Progress Electromagn. Res. Symp., Tokyo, Japan, 2006, pp. 256-260.

[3] E. Zastrow, S. Davis, and S. Hagness, "Safety assessment of breast cancer detection via ultrawideband microwave radar operating in pulsed-radiation mode," Microw. Opt. Technol. Lett., vol. 49, no. 1, pp. 221-225, 2007.

[4] P. Meaney, M. Fanning, D. Li, S. Poplack, and K. Paulsen, "A clinical prototype for active microwave imaging of the breast," IEEE Trans. Microw. Theory Tech., vol. 48, no. 11, pp. 1841-1853, Nov. 2000.

[5] P. Meaney, M. Fanning, T. Raynolds, C. Fox, Q. Fang, C. Kogel, S. Poplack, and K. Paulsen, "Initial clinical experience with microwave breast imaging in women with normal mammography," Acad. Radiol., vol. 14, no. 2, pp. 207-218, 2007.

[6] M. Lazebnik, D. Popovic, L. McCartney, C. Watkins, M. Lindstrom, J. Harter, S. Sewall, T. Ogilvie, A. Magliocco, T. Breslin et al., "A large-scale study of the ultrawideband microwave dielectric properties of normal, benign and malignant breast tissues obtained from cancer surgeries," Phys. Med. Biol., vol. 52, no. 20, pp. 6093-6115, 2007.

[7] J. Sill and E. Fear, "Tissue sensing adaptive radar for breast cancer detection-Experimental investigation of simple tumor models," IEEE Trans. Microw. Theory Tech., vol. 53, no. 11, pp. 3312-3319, Nov. 2005. 
[8] E. Fear, J. Sill, and M. Stuchly, "Experimental feasibility study of confocal microwave imaging for breast tumor detection," IEEE Trans. Microw. Theory Tech., vol. 51, no. 3, pp. 887-892, Mar. 2003.

[9] D. Kurrant, E. Fear, and D. Westwick, "Tumor response estimation in radar-based microwave breast cancer detection," IEEE Trans. Biomed. Eng., vol. 55, no. 12, pp. 2801-2811, Dec. 2008. DOI: 10.1109/TBME.2008.921164.

[10] E. Bond, X. Li, S. Hagness, and B. Van Veen, "Microwave imaging via space-time beamforming for early detection of breast cancer," IEEE Trans. Antennas Propag., vol. 51, no. 8, pp. 1690-1705, Aug. 2003.

[11] X. Li, S. Davis, S. Hagness, D. van der Weide, and B. Van Veen, "Microwave imaging via space-time beamforming: Experimental investigation of tumor detection in multilayer breast phantoms," IEEE Trans. Microw. Theory Tech., vol. 52, no. 8, pp. 1856-1865, Aug. 2004.

[12] S. Davis, B. Van Veen, S. Hagness, and F. Kelcz, "Breast tumor characterization based on ultrawideband microwave backscatter," IEEE Trans. Biomed. Eng., vol. 55, no. 1, pp. 237-246, Jan. 2008.

[13] P. Kosmas and C. Rappaport, "FDTD-based time reversal for microwave breast cancer detection localization in three dimensions," IEEE Trans. Microw. Theory Tech., vol. 54, no. 4, Part 2, pp. 1921-1927, Apr. 2006.

[14] D. Li, P. Meaney, T. Raynolds, S. Pendergrass, M. Fanning, and K. Paulsen, "Parallel-detection microwave spectroscopy system for breast imaging," Rev. Sci. Instrum., vol. 75, no. 7, pp. 2305-2313, 2004.

[15] F. Qianqian, P. Meaney, S. Geimer, A. Streltsov, and K. Paulsen, "Microwave image reconstruction from 3-D fields coupled to 2-D parameter estimation," IEEE Trans. Med. Imag., vol. 23, no. 4, pp. 475-484, Apr. 2004.

[16] S. Poplack, T. Tosteson, W. Wells, B. Pogue, P. Meaney, A. Hartov, C. Kogel, S. Soho, J. Gibson, and K. Paulsen, "Electromagnetic breast imaging: Results of a pilot study in women with abnormal mammograms," Radiology, vol. 243, no. 2, pp. 350-359, 2007.

[17] T. Takenaka, T. Tanaka, H. Harada, and S. He, "FDTD approach to timedomain inverse scattering problem for stratified lossy media," Microw. Opt. Technol. Lett., vol. 16, no. 5, pp. 292-296, 1997.

[18] T. Tanaka, T. Takenaka, and S. He, "An FDTD approach to the timedomain inverse scattering problem for an inhomogeneous cylindrical object," Microw. Opt. Technol. Lett., vol. 20, no. 1, pp. 72-77, 1999.

[19] T. Takenaka, H. Jia, and T. Tanaka, "Microwave imaging of electrical property distributions by a forward-backward time-stepping method," $J$. Electromagn. Waves Appl., vol. 14, no. 12, pp. 1609-1626, 2000.

[20] H. Zhou, T. Takenaka, and T. Tanaka, "Time-domain reconstruction of lossy objects using dipole antennas," Microw. Opt. Technol. Lett., vol. 44, pp. 238-243, 2005.

[21] J. Johnson, H. Zhou, and T. Takenaka, "Experimental three-dimensional time-domain reconstruction of dielectric objects for breast cancer detection," in Proc. Mediterranean Microw. Symp., 2006, pp. 423-426.

[22] J. Johnson, T. Takenaka, and T. Tanaka, "Two-dimensional time-domain inverse scattering for quantitative analysis of breast composition," IEEE Trans. Biomed. Eng., vol. 55, no. 8, pp. 1941-1945, Aug. 2008.

[23] K. Hong Ping, T. Moriyama, T. Takenaka, and T. Tanaka, "Reconstruction of breast composition in a free space utilizing 2-D forward-backward time-stepping for breast cancer detection," in Proc. 4th IET Int. Conf. Adv. Med., Signal Inform. Process. (MEDSIP'08), pp. 1-4.

[24] M. Gustafsson and S. He, "A wave-splitting based optimization approach to multi-dimensional time-domain electromagnetic inverse problems," Math. Comput. Simul., vol. 50, no. 5-6, pp. 541-551, 1999.

[25] I. Rekanos and A. Raisanen, "Microwave imaging in the time domain of buried multiple scatterers by using an FDTD-based optimization technique," IEEE Trans. Magn., vol. 39, no. 3, pp. 1381-1384, May 2003.

[26] D. W. Winters, E. J. Bond, B. D. Van Veen, and S. C. Hagness, "Estimation of the frequency-dependent average dielectric properties of breast tissue using a time-domain inverse scattering technique," IEEE Trans. Antennas Propag., vol. 54, no. 11, pp. 3517-3528, Nov. 2006.

[27] A. Fhager, P. Hashemzadeh, and M. Persson, "Reconstruction quality and spectral content of an electromagnetic time-domain inversion algorithm," IEEE Trans. Biomed. Eng., vol. 53, no. 8, pp. 1594-1604, Aug. 2006.

[28] V. Hutson and J. Pym, Applications of Functional Analysis and Operator Theory. London, U.K.: Academic, 1980.

[29] R. Fletcher, Practical Methods of Optimization. New York: WileyInterscience, 1987.

[30] M. Converse, E. Bond, B. Veen, and S. Hagness, "A computational study of ultra-wideband versus narrowband microwave hyperthermia for breast cancer treatment," IEEE Trans. Microw. Theory Tech., vol. 54, no. 5, pp. 2169-2180, May 2006
[31] A. Taflove and S. Hagness, Computational Electrodynamics: The FiniteDifference Time-Domain Method, 3rd ed. Norwood, MA: Artech, 2005.

[32] P. Kosmas, C. Rappaport, and E. Bishop, "Modeling with the FDTD method for microwave breast cancer detection," IEEE Trans. Microw. Theory Tech., vol. 52, no. 8, pp. 1890-1897, Aug. 2004.

[33] D. Winters, J. Shea, E. Madsen, G. Frank, B. Van Veen, and S. Hagness, "Estimating the breast surface using UWB microwave monostatic backscatter measurements," IEEE Trans. Biomed. Eng., vol. 55, no. 1, pp. 247-256, Jan. 2008.

[34] T. Williams, J. Sill, and E. Fear, "Breast surface estimation for radarbased breast imaging systems," IEEE Trans. Biomed. Eng., vol. 55, no. 6, pp. 1678-1686, Jun. 2008.

[35] T. Williams, E. Fear, and D. Westwick, "Tissue sensing adaptive radar for breast cancer detection - investigations of an improved skin-sensing method," IEEE Trans. Microw. Theory Tech., vol. 54, no. 4, pp. 13081314, Apr. 2006.

[36] M. Lazebnik, M. Okoniewski, J. Booske, and S. Hagness, "Highly accurate Debye models for normal and malignant breast tissue dielectric properties at microwave frequencies," IEEE Microw. Wireless Compon. Lett., vol. 17, no. 12, pp. 822-824, Dec. 2007.

[37] S. Hagness, A. Taflove, and J. Bridges, "Three-dimensional FDTD analysis of a pulsed microwave confocal system for breast cancer detection: Design of an antenna-array element," IEEE Trans. Antennas Propag., vol. 47, no. 5, pp. 783-791, May 1999.

[38] P. Meaney, Q. Fang, and K. Paulsen, "Data collection strategies and their impact on 3-D microwave imaging of the breast," in Proc. IEEE Antennas Propag. Soc. Int. Symp., 2005, vol. 1b, pp. 183-186.

[39] M. Bertero, "Linear inverse and ill-posed problems," Adv. Electron. Electron Phys., vol. 75, no. 1, pp. 1-120, 1989.

[40] A. D. Wiles, D. G. Thompson, and D. D. Frantz, "Accuracy assessment and interpretation for optical tracking systems," in Proc. SPIE Conf., May 2004, vol. 5367, pp. 421-432.

[41] T. Moore, M. Lunt, B. McManus, M. Anderson, and A. Herrick, "Seventeen-point dermal ultrasound scoring system-a reliable measure of skin thickness in patients with systemic sclerosis," Rheumatology, vol. 42, no. 12, pp. 1559-1563, 2003.

[42] T. Tanaka, N. Kuroki, and T. Takenaka, "Filtered forward-backward timestepping method applied to reconstruction of dielectric cylinders," $J$. Electromagn. Waves Appl., vol. 17, no. 2, pp. 253-270, 2003.

[43] P. Lobel, L. Blanc-Feraud, C. Pichot, and M. Barlaud, "A new regularization scheme for inverse scattering," Inverse Problems, vol. 13, no. 2, pp. 403-410, 1997.

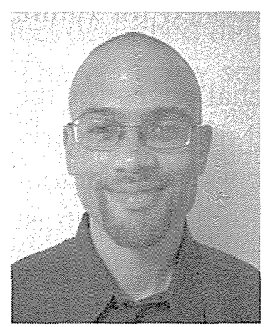

Jessi E. Johnson (S'98-M'04) received the B.S. degree in electrical engineering and the Ph.D. degree in electrical engineering ( $R F$ and microwave circuit design) from the University of California, Davis, in 1999 and 2004.

From 2004 to 2005, he was a Postdoctoral Fellow at the Microwave Hyperthermia Laboratory, University of California, San Francisco. From 2005 to 2007, he was a Visiting Research Fellow with Dr. Takashi Takenaka's Research Group, Nagasaki University, Nagasaki, Japan, where he was engaged in the investigation of microwave imaging systems for biomedical applications. He is currently a Senior Research Scientist at Miramar Labs, Inc., Sunnyvale, CA.

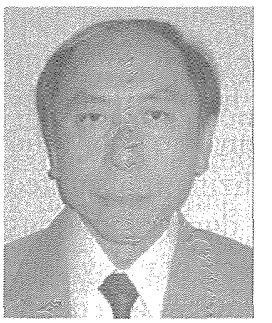

Takashi Takenaka (M'89) received the B.E., M.E., and D.E. degrees from Kyushu University, Fukuoka, Japan, in 1973, 1975, and 1979, respectively, all in communication engineering.

In 1978, he joined the Department of Computer Science and Communication Engineering, Kyushu University. In 1989, he was with Nagasaki University, Nagasaki, Japan, where he is currently a Professor with the Department of Electrical and Electronic Engineering. His current research interests include direct/inverse scattering problems and complex ma- 


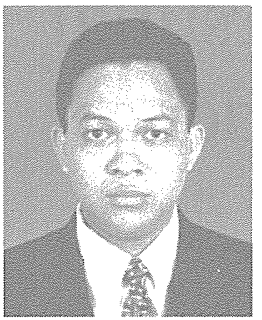

Kismet Anak Hong Ping was born in Kuching, Malaysia, on July 24, 1974. He received the B.Eng. (Hons.) degree in electronics and telecommunication engineering from the University Malaysia Sarawak, Kota Samarahan, Malaysia, and the M.Sc. degree in digital communication systems from Loughborough University, England, U.K., in 1998 and 2000, respectively. He is currently working toward the Ph.D. degree from the Graduate School of Science and Technology, Nagasaki University, Nagasaki, Japan.

In 2000, he joined the Department of Electronics Engineering, University Malaysia Sarawak, as a Lecturer. His current interests include electromagnetic direct/inverse scattering, optimization techniques for microwave imaging, wave propagation, computational electromagnetic, and antenna design.

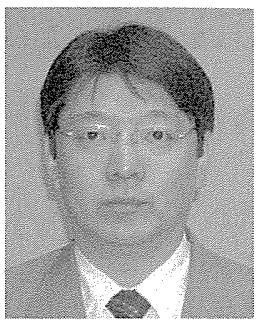

Toshiyuki Tanaka was born in Fukuoka, Japan, on March 24, 1962. He received the B.E. degree in electrical engineering from Nagasaki University, Nagasaki, Japan, in 1984, and the M.E. and D.E. degrees in communication engineering from Kyushu University, Fukuoka, Japan, in 1986 and 1989, respectively.

From 1989 to 2000, he was with the Department of Electrical Engineering and Computer Science, Nagasaki University, where he is currently an Associate Professor with the Division of System Science, Graduate School of Science and Technology. His current research interests include direct and inverse scattering problems, and numerical analysis.

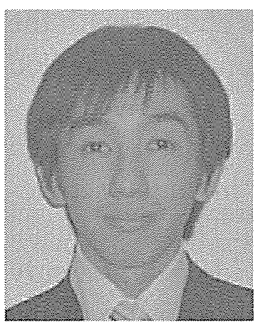

Shunsuke Honda received the B.E. and M.E. degrees in electrical and electronic engineering from Nagasaki University, Nagasaki, Japan, in 2007 and 2009 , respectively.

His current research interests include parallel computations on inverse scattering problems. 